\title{
Micro Thermal Shear Stress Sensor with and without Cavity underneath
}

\author{
Jin-Biao Huang, Chih-Ming Ho, Steve Tung, Chang Liu ${ }^{*}$, and Yu-Chong Tai ${ }^{*}$ \\ Mechanical, Aerospace, and Nuclear Engineering Department \\ University of California, Los Angeles \\ Los Angeles, CA 90095-1597 \\ *Electrical Engineering Department \\ California Institute of Technology \\ Pasadena, CA 91125 \\ Phone (310) 825-8275 Fax (310) 206-4830 E-Mail: jinbiao@seas.ucla.edu
}

\begin{abstract}
Micro hot-film shear-stress sensors have been designed and fabricated by surface micromachining technology compatible with IC technology. A poly-silicon strip, $2 \mu \mathrm{mx80} \mu \mathrm{m}$, is deposited on the top of a thin silicon nitride film and functions as the sensor element. By using sacrificial-layer technique, a cavity (vacuum chamber), $200 \times 200 \times 2 \mu \mathrm{m}^{3}$, is placed between the silicon nitride film and silicon substrate. This cavity significantly decreases the heat loss to the substrate. For comparison purposes, a sensor structure without a cavity has also been designed and fabricated on the same chip. Theoretical analyses for the two vertical structures with and without a cavity show that the former has a lower frequency response and higher sensitivity than the latter. When the sensor is operated in constant temperature mode, the cut-off frequencies can reach $130 \mathrm{k}-\mathrm{Hz}$ and $9 \mathrm{k}-\mathrm{Hz}$ respectively for the sensors without and with cavities.
\end{abstract}

\section{INTRODUCTION}

Fluid flow over a solid boundary generates a velocity gradient that produces a shear stress at the wall. This wall shear stress is one of the important parameters in fluid mechanics. There are many measurement techniques to determine shear stress [1]. One common technique is a flushmounted hot-wire/hot-film anemometer. With micromachining technology, more advanced techniques are available [4-6]. The hot-film anemometer is an example of an indirect shear-stress measurement device. The heat transfer from a resistively heated element to the flowing fluid is measured, from which a value for shear stress is inferred. Although this type of probe has been employed widely for shear stress measurements, the factors influencing their unsteady performance are not yet well understood [1-2]. It has been well accepted that the thermal properties of the substrate affect sensor operation [2-3]. Some workers have attempted to isolate the sensor from the substrate[7-8], however, none of these efforts resulted in the desired high frequency responses. The effect of the insulation on the sensor's performance remains unknown systematically. A finite difference method was provided to solve the one dimensional heat diffusion equation for the frequency response of a hot film sensor [3]. The different sensor geometries and insulation materials were used to experimentally study the sensor and its relationship with the substrate [2]. No fully theoretical computations and experimental efforts are known to the authors.

In this work, two different kinds of hot-film shear-stress sensors have been designed and fabricated by micromachining technology: one with a vacuum chamber (cavity) underneath to insulate it from the substrate, and the other one without. Except for the vertical structure, these two sensors have identical geometries. Heat transfer models have been developed for the sensors and the electric analogy of the heat transfer was also used. Both analytical solution and experimental results have been obtained. The micromachined hot-film sensors were originally developed for a distributed micro-electro-mechanical system (MEMS) for viscous drag reduction research [3].

\section{SENSOR AND STRUCTURE}

Fig. 1 schematically shows the top and cross-sectional views of the micro hot-film sensor with a cavity underneath. By using micromachining technology, a vacuum chamber (cavity), the square part (dashed line) in the Fig. 1(a), is placed under a thin silicon nitride film. The size of the cavity is $200 \times 200 \times 2 \mu \mathrm{m}^{3}$. A poly-silicon strip, $2 \times 80 \mu \mathrm{m}^{2}$, is deposited on the silicon nitride film and functions as the sensor element. The metal leads connect the sensor element to outside through bonding pads. The other sensor without a cavity underneath is also included on the same chip. Both sensors, with and without cavity, have identical geometries. The flow direction is perpendicular to the long side of the sensor strip.

The cavity is formed by sacrificial-layer (PSG, phosphosilicate glass) technique. Both silicon nitride and poly-silicon layers are deposited by LPCVD (low pressure chemical vapor deposition). The aluminum metalization forms the metal leads. The poly-silicon resistor is uniformly doped to a low sheet-resistance of typically $50 \Omega / \square$. The measured TCR (temperature coefficient of resistivity) of the sensor at this doping level is about $0.09 \% /{ }^{\circ} \mathrm{C}$. The detailed process steps are given in [4]. The sensor with negative TCR at lower doping level will be given at some other place.

A photograph of the microfabricated sensors is shown in Fig. 2, in which the left one is the sensor with a cavity and 
the right one without a cavity. The white parts in the figure are metal leads due to the optical reflection. Although the cavity is not shown thoroughly, its frame still can be seen clearly. The theoretical models for these sensors will be given in the following section.

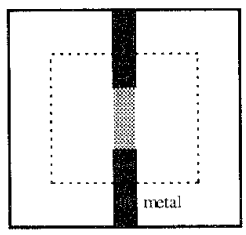

(a)

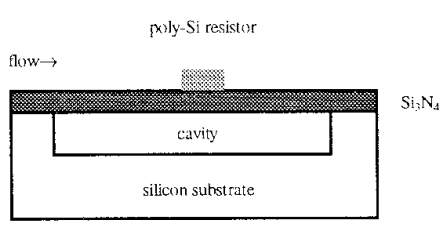

(b)
Fig. 1. Schematic top (a) and cross-sectional (b) views of the micro hot-film shear-stress sensor

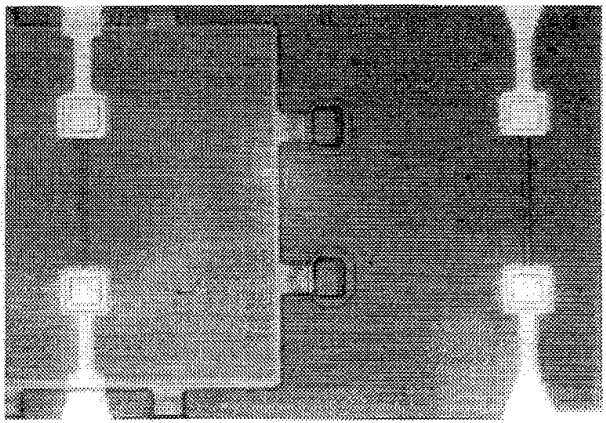

Fig. 2. The photograph of the micrfabricated hot-film shear-stress sensors with a cavity (the left) and without a cavity (the right) underneath.

\section{THEORETICAL MODELS}

A general three-layer structure is used to characterize the dynamic performances in the micro sensors, as shown in Fig. 3(a). The film layer at the top represents the sensor element. The insulation layer represents the silicon nitride diaphragm. The silicon substrate with large thermal conductivity is treated as a heat sink. The length and width of the sensor is $L$ and $W$. The $q$ in the figure represent heat transfer. The subscripts $f, i$, and $c$ represent the heat transfer to film, insulation layer, and convective heat transfer to the measured fluid, respectively. The same subscripts are used for layer thickness $d$ and other thermal parameters in following paragraphs. The case of the sensor with a cavity underneath can be considered as that the heat transfer $q_{\mathrm{i}}$ goes laterally first and then goes vertically to the heat sink (substrate) as shown in Fig. 3 (b).

The energy balance equation in the general structure of the Fig. 3 (a) can be given as follows:

$$
i^{2} R=c_{f} m_{f} \frac{d T_{f}}{d t}+c_{i} m_{i} \frac{d T_{i}}{d t}+h\left(u_{\tau}\right) A\left(T-T_{o}\right)
$$

The heating current $i$ through the resistive sensor $R$ produces heating power $i^{2} R$. The power is dissipated or stored in the following three ways, corresponding to the three terms on the right-hand side of above equation. 1) convective heat transfer $q_{\mathrm{c}}=h\left(u_{\tau}\right) A\left(T-T_{0}\right)$ to the sensor's environment, in which $T$ and $T_{0}$ are respectively the temperatures of the sensor element and of the heat sink. The convective heat transfer coefficient is represented by $h\left(u_{\tau}\right)$ and it is a function of shear velocity $u_{\tau}$. The relation between the shear velocity $u_{\tau}$ and the wall shear stress $\tau_{\mathrm{w}}$ is $\tau_{\mathrm{w}}=u_{\tau}^{2} \rho$ where $\rho$ is the measured fluid density. The heat transfer going through the insulation layer, substrate and then to the measured environment is also included in this term. This makes the effective heat transfer area $A$ a little bit larger than $W \times L .2$ ) conductive heat transfer $q_{\mathrm{f}}$ to the film. It equals the energy stored in the film, $q_{\mathrm{f}}=c_{\mathrm{f}} m_{\mathrm{f}} d T_{\mathrm{f}} / d t$, where $c_{\mathrm{f}}, m_{\mathrm{f}}$, and $T_{\mathrm{f}}$ are the specific heat, mass, and temperature of the film layer, respectively. 3) conductive heat transfer to the insulation layer and stored in it, $q_{\mathrm{i}}=c_{\mathrm{i}} m_{\mathrm{i}} d T_{\mathrm{i}} / d t$, with the same parameter notation for the insulation layer as in the film case. The last two conductive heat transfer terms have the following relations to thermal conductivity.

$$
\begin{aligned}
& q_{i}=c_{i} m_{i} \frac{d T_{i}}{d t}=\frac{k_{i} A\left(T_{f}-T_{i}\right)}{d_{i}} \\
& q_{f}+q_{i}=\frac{k_{f} A\left(T-T_{f}\right)}{d_{f}}
\end{aligned}
$$

where $k_{\mathrm{i}}$ and $k_{\mathrm{f}}$ are the thermal conductivities of insulation and film layers, respectively.
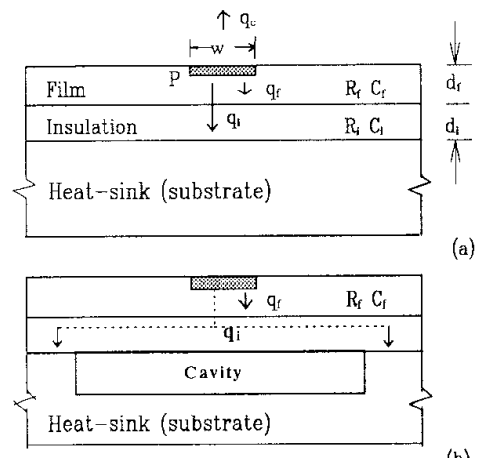

(a)

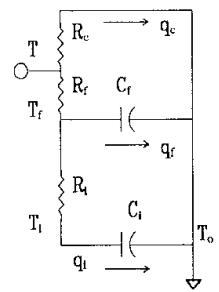

(c)

(b)

Fig. 3. The heat transfer models for the general sensor with an insulation layer underneath (a), the sensor with a cavity underneath (b), and their electric-analogy's equivalent circuit (c).

Considering the fluctuating time dependent part of each above variable, neglecting secondary terms, and using a Laplace transform, the transfer function between the sensor's temperature and the input variable $\Delta F$ can be obtained as follows:

$$
\begin{aligned}
& \frac{\Delta T}{\Delta F}=\left(\frac{\alpha_{R}}{\alpha i^{2} R_{o}}\right) \frac{t_{2} s+1}{t_{1} t_{2} s^{2}+\left(t_{1}+t_{2}+t_{3}\right) s+1} \\
& \Delta F=\frac{\partial P}{\partial i} \Delta I-\left(\bar{T}-T_{o}\right) A \Delta H
\end{aligned}
$$

where $\Delta F$ is the Laplace transform form of the input variable, which is a function of both electrical input 
perturbation $\Delta I$ and shear-stress related input perturbation $\Delta H, \bar{T}$ is the static temperature of the sensor. $\alpha_{\mathrm{R}}, \alpha$, and $R_{\mathrm{o}}$ in (4) are resistive overheat ratio, temperature coefficient of resistivity (TCR) of the sensor element, and sensor resistance at temperature $T_{0}$, respectively. The time contents $t_{1}, t_{2}$, and $t_{3}$ in (4) are

$$
\begin{aligned}
& t_{f}=\frac{\alpha_{R}}{i^{2} \alpha R_{0}} c_{f} m_{f} \\
& t_{2}=\frac{d_{i}}{k_{i} A} c_{i} m_{i} \\
& t_{3}=\frac{\alpha_{R}}{i^{2} \alpha R_{0}} c_{i} m_{i}
\end{aligned}
$$

Although the system shown in (4) is second order, the pole $s_{1} \approx-1 /\left(t_{1}+t_{2}+t_{3}\right)$ is dominant. So the system behaves just like first order system and its time constant is

$$
t=t_{1}+t_{2}+t_{3}
$$

This total time constant consists of three parts, the first part $t_{1}$ is exactly the same as the time constant previous workers found [9], the last two parts $t_{2}$ and $t_{3}$ obtained here indicate how the insulation layer parameters affect the sensors' dynamic performances. From (6) and (7) it can be seen that the existence of the insulation layer tends to enlarge the time constant and to decrease the frequency response of the system. The smaller the thermal conductivity $k_{\mathrm{i}}$ of the insulation layer, the bigger the effect. The larger the specific heat $c_{\mathrm{i}}$ and mass $m_{\mathrm{i}}$ of the insulation layer, the longer the time constant. This also answers why people can't get high dynamic performance by insulating the sensor from the substrate.

For the sensor with a cavity underneath, the heat transfer goes laterally first in the insulation layer and then vertically to the heat sink. This makes the time constant $t_{2}$ change to

$$
t_{2}=\frac{L_{c}}{2 k_{i} d_{i} L} c_{i} m_{i}
$$

where $L_{\mathrm{c}}$ is the half length of the cavity side and ' 2 ' in the denominator is due to bi-directional heat transfer in the insulation layer. $t_{2}$ and $t_{3}$ remain the same. Since $L_{c}$ in (8) is much bigger than $d_{\mathrm{i}}$ in (6) while $2 d_{\mathrm{i}} L$ in (8) and $A$ in (6) are on the same order, the time constant $t_{2}$ of the sensor with a cavity underneath is longer than that without a cavity. Actually the effective heat transfer area $A$ and effective masses $m_{\mathrm{f}}$ and $m_{\mathrm{i}}$ are also increased because more volume is heated due to the existence of the cavity. All these factors tend to make the total time constant $t$ of the sensor with a cavity longer than that without a cavity.

It should be mentioned that $\mathrm{Si}$ substrate is not an ideal heat-sink. The part of the substrate behaves just like the insulation layer. This will make the actual time constant a little bit longer than that indicated by (6), (7), and (8).

The sensitivity of the system can also be obtained based on (4). We can combine (4) and (5) as follows by replacing $\Delta l=0$ and $\Delta H=1 / \mathrm{s}$ into (5):

$$
\Delta T=-\frac{\alpha_{R} A\left(\bar{T}-T_{o}\right)}{\alpha i^{2} R_{o}} \frac{\left(t_{2} s+1\right)(1 / s)}{t_{1} t_{2} s^{2}+\left(t_{1}+t_{2}+t_{3}\right) s+1}
$$

This equation can be written in the following form too:

$$
\Delta T=\frac{K_{o}}{s}+\frac{K_{1}}{\left(s-s_{1}\right)}+\frac{K_{2}}{\left(s-s_{2}\right)}
$$

where $s_{1}$ and $s_{2}$ are two poles of the system, $K_{0}, K_{1}$, and $K_{2}$ are constants. From (9) and (10), we can get sensitivity constant $K_{0}$

$$
K_{0}=\left.s \Delta T\right|_{s=0}=-\frac{\alpha_{R} A\left(\bar{T}-T_{o}\right)}{\alpha i^{2} R_{o}}
$$

In comparison with (6) and (7), the sensitivity has a conflict requirement with system's time constant.

Alternatively, the electric analogy of the heat transfer can be used and the equivalent circuit is shown in Fig. 3 (c). The quantity $d / A k$ in (2) and (3) is equivalent to a thermal resistance $R_{\mathrm{i}}$ for the insulation layer or $R_{\mathrm{f}}$ for the film layer. Similarly, the quantity $\mathrm{cm}$ in (1) is equivalent to a thermal capacitance $C_{\mathrm{i}}$ or $C_{\mathrm{f}}$. The same result with clearer physical meaning can be obtained by using this method, e.g., $t_{2}=R_{\mathrm{i}} C_{\mathrm{i}}$.

\section{EXPERIMENTS}

After the fabrication of the hot-film sensor, Both CC (constant current) and CT (constant-temperature) circuits have been designed for sensor operation. Here we focus on the CT circuit as shown in Fig. 4. In the figure, $R$ is the sensor, $R_{\mathrm{oh}}$ for adjusting resistive overheat ratio, $R_{\mathrm{c}}$ and $C_{\mathrm{c}}$ are for compensation. Since suitable velocity fluctuations are not readily available, one usually relies on electronic test signals. According to both theoretical analyses[10] and experimental confirmation[2], the frequency response or time constant can be obtained by feeding an electronic sine wave or square wave into the CT circuit. The terminal $E_{\mathrm{t}}$ in Fig. 4 is for this purpose.

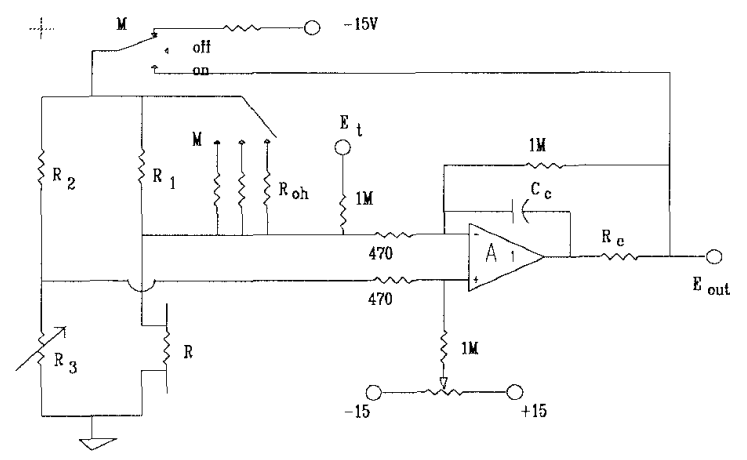

Fig. 4. The constant-temperature circuit for the hot-film shear-stress sensor in which $R$ is the sensor and $E_{\mathrm{t}}$ is the terminal for the testing signal.

The square wave responses of both sensors with and without a cavity are measured and shown in Figs. 5 (a) and (b), respectively. The measured time constants are $72 \mu$ s and $5.2 \mu \mathrm{s}$ for the former and the latter, respectively. The 
difference is clear and the latter (without a cavity) has much shorter time constant (one order of magnitude). This is consistent with the results indicated by theoretical models in section III. An overheat ratio of $5.5 \%$ is used in this experiment. According to the approximate relation between time constant $t_{\mathrm{c}}$ and cut-off frequency $f_{\mathrm{c}}$ for $\mathrm{CT}$ operation, $f_{\mathrm{c}}=1 /\left(1.5 t_{\mathrm{c}}\right)$, the cut-off frequency can be estimated. These frequencies are $9 \mathrm{k}-\mathrm{Hz}$ and $128 \mathrm{k}-\mathrm{Hz}$ for the sensors with cavity and without cavity, respectively, based on the time constant measurements.

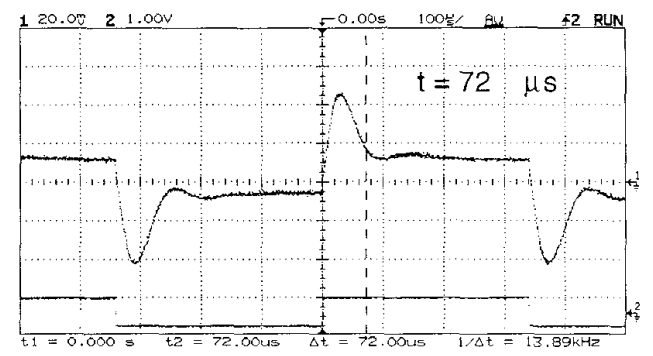

(a)

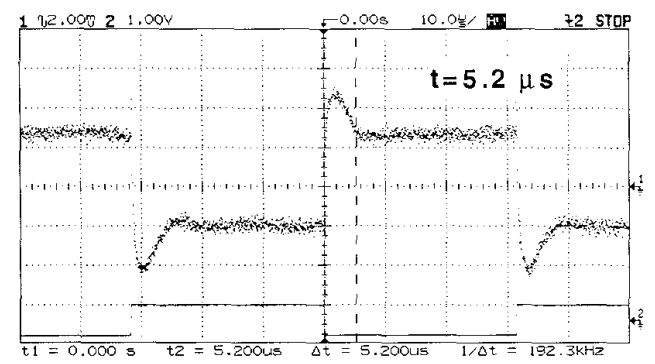

(b)

Fig. 5. The square wave responses of the hot-film sensors with (a) and without (b) a cavity underneath. The time constants are $72 \mu$ s and $5.2 \mu$ s for the top one and the bottom one, respectively.

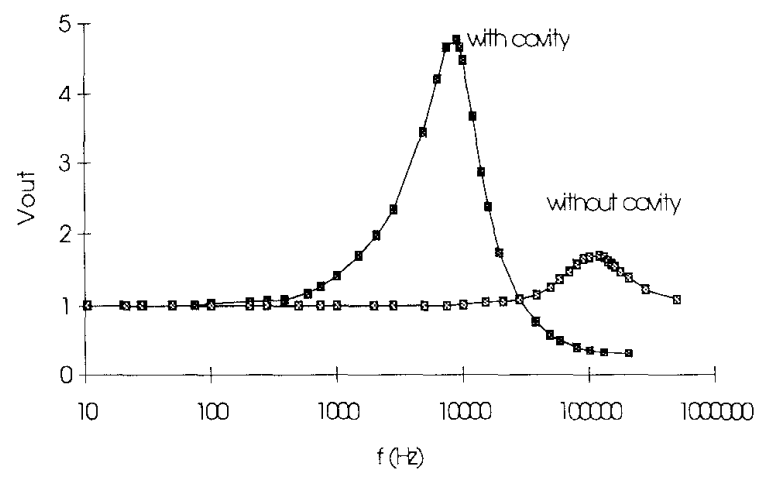

Fig. 6. The frequency responses of the micro hot-film sensors with and without cavity. The cut-off frequencies are 9 kilo- $\mathrm{Hz}$ and $130 \mathrm{kilo}-\mathrm{Hz}$, for the former and the latter, respectively.

The real frequency responses have been obtained by using an electronic sine wave instead of square wave. Fig. 6 presents the results for both sensors with and without a cavity. The cut-off frequencies are respectively 9 and $130 \mathrm{k}$ $\mathrm{Hz}$. This is consistent with the estimates based on time constant measurements.

In Fig. 5 the vertical scales are $20 \mathrm{mV} /$ div. and $2 \mathrm{mV} /$ div. for the sensors with a cavity(a) and without a cavity(b), respectively. So the sensitivity of the former is about one order of magnitude greater than the latter. This is also confirmed by wind tunnel measurements.

\section{CONCLUSIONS}

The hot-film shear-stress sensors with and without a cavity underneath have been designed and fabricated by micromachining technology. The theoretical heat transfer models and analytical solutions indicate that the insulation layer has an important effect to the sensors' frequency responses. Both theoretical and experimental results show that the sensor with a cavity underneath has a slower frequency response and higher sensitivity in comparison with the sensor without a cavity.

\section{ACKNOWLEDGMENT}

This work is supported by AFOSR URI project (University Research Initiative).

\section{REFERENCES}

[1] T. J. Hanratty and J. A. Cambell, "Measurement of wall shear stress," in Fluid Mechanics Measurements, R. J. Goldstein, Ed. New York: Hemisphere, 1983, pp. 559-615.

[2] M. J. Moen and S. P. Schneider, "The effect of sensor size and substrate properties on the performance of flush-mounted hot-film sensors," in Thermal Anemometry-1993, D. E. Stock, S. A. Sherif, A J. Smits, and J. Davidson, Eds. ASME, 1993, pp. 249-261.

[3] S. Albin, A. Bulusu, S. D. Martinson, and D. S. Gray, "Frequency response simulations of a diamond based sensor for supersonic flows," ibid., 1993, pp. 181-184.

[4] C. Liu, Y.C. Tai, J.-B. Huang, and C.-M. Ho, "Surface micromachined thermal shear stress sensor," ASME Symposium on Application of Microfabrication to Fluid Mechanics, Chicago, IL, 1994, pp. 9-11.

[5] J. Shajii, K.-Y. Ng, and M. A. Schmidt, "A microfabricated floatingelement shear stress sensor using wafer-bonding technology," IEEE/ASME J. of Microelectromechanical Systems, vol. 1, no. 2, pp. 89-94, 1992.

[6] M. A. Schmidt, R. T. Howe, S. D. Senturia, and J. H. Haritonidis, "Design and calibration of a microfabricated floating-element shearstress sensor," IEEE Trans. Electron Devices, vol. 35, no. 6, pp. 750 757,1988

[7] C. O. Ajagu and P. A. Libby, "Modified gauge for time-resolved skinfriction measurements," Review of Scientific Instruments, vol. 53, no. 12, pp. 1920-1926, 1982.

[8] D. Reda, "Rise-time response of nickel-foil-on-Kapton-substrate, hotfilm shear stress sensors," AIAA Paper 91-0169, 1991.

[9] R. F. Blackwelder, "Hot-wire and hot-film anemometers," in Methods of Experimental Physics: Fluid Dynamics, R. J. Emrich, Ed., Academic Press, 1981, pp. 259-314.

[10] P. Freymuth, "Frequency response and electronic testing for constanttemperature hot-wire anemometers," $\mathbf{J}$. of Physics E: Scientific Instruments, vol. 10, pp. 705-710, 1977. 